This Technical Report is also published as Chapter 17 IN

M-Health: Emerging Mobile Health Systems, Robert H. Istepanian, Swamy Laxminarayan, Constantinos S. Pattichis, Editors, 2006.

Springer. $624 \mathrm{p}$.

ISBN: 0-387-26558-9.

As

\title{
MOBIHEALTH: MOBILE SERVICES FOR HEALTH PROFESSIONALS
}

Val Jones*, Aart van Halteren, Nikolai Dokovsky, George Koprinkov, Jan Peuscher, Richard Bults, Dimitri Konstantas, Ing Widya and Rainer Herzog.

* Val Jones, Centre for Telematics and Information Technology/Faculty of Electrical Engineering, Mathematics and Computer Science, University of Twente, PO Box 217, 7500 AE, Enschede, The Netherlands, v.m.jones@ewi.utwente.nl. 


\title{
MOBIHEALTH: MOBILE SERVICES FOR HEALTH PROFESSIONALS
}

\author{
Val Jones*, Aart van Halteren, Nikolai Dokovsky, \\ George Koprinkov, Jan Peuscher, Richard Bults, Dimitri \\ Konstantas, Ing Widya and Rainer Herzog.
}

\section{INTRODUCTION}

The concept behind the MobiHealth project (MobiHealth, 2002). was to bring together the technologies of Body Area Networks (BANs), wireless broadband communications and wearable devices to provide mobile healthcare services for patients and health professionals. For patients, these technologies enable remote patient care services such as management of chronic conditions and detection of health emergencies. For health professionals the technology offers access to information and communication services from a mobile device, thus enabling mobility for the individual professional and supporting the operation of distributed 'virtual' healthcare teams (sensu Pitsillides, 1999).

During the project a generic BAN for healthcare and a generic mhealth service platform were developed, trialled and evaluated. The main devices used in patient BANs were medical sensors. Biosignals measured by sensors connected to the BAN were transmitted to a remote healthcare location over $2.5 / 3 \mathrm{G}$ public wireless networks, using commercial GPRS services and test infrastructures for UMTS. The MobiHealth BAN and service platform were trialled in Spain, The Netherlands, Sweden and Germany.

The overall goal of MobiHealth was to evaluate the ability of 2.5 and 3G communication technologies to support innovative mobile health services. The main output of the project therefore is an assessment of the suitability of GPRS and UMTS to support such services.

\footnotetext{
*Val Jones, Centre for Telematics and Information Technology/Faculty of Electrical Engineering, Mathematics and Computer Science, University of Twente, PO Box 217, 7500 AE, Enschede, The Netherlands, v.m.jones@ewi.utwente.nl.
} 


\section{MOBIHEALTH: MOBILE SERVICES FOR HEALTH PROFESSIONALS}

The MobiHealth concept arose out of earlier work at the University of Twente (Jones et al., 1999; Jones 2001, Jones et al., 2001b; Jones et al., 2001c; WWRF, 2001). The MobiHealth consortium involving 14 European partners was brought together during 2001. The project began on May $1^{\text {st }} 2002$ and was funded by the European Commission under IST FP5 and has been reported in (Jones et al, 2001a; Konstantas et al., 2002a,b; Widya et al., 2003, Dokovsky et al., 2003).

This remainder of this chapter presents the MobiHealth system (Section 2), MobiHealth services from the health professional point of view (Section 3), and some of the issues and future challenges raised by the project (Section 4).

\section{THE MOBIHEALTH SYSTEM}

The components of the MobiHealth system are presented below. The software was developed at the University of Twente and is available as Open Source software.

\subsection{Body Area Networks}

The concept of the Body Area Networks has been developed in different directions since the original work at IBM (Zimmerman, 1999), eg. by (van Dam, 2001; Jones et al., 2001a; Jones et al., 2001b; Schmidt, 2001). In our definition, a BAN is "a collection of (inter) communicating devices which are worn on the body, providing an integrated set of personalised services to the user" (WWRF, 2001). A BAN can be thought of as a computer network which is worn on the body and which moves around with the person.

A BAN incorporates a set of devices which perform specific functions and which also perform communication, perhaps via a central controlling device. Communication amongst the elements of a BAN is called intra$B A N$ communication. Intra-BAN communication may be carried over a wired or a wireless medium. If the BAN communicates externally, i.e. with other networks, this communication is referred to as extra-BAN communication. Extra-BAN communication is usually mediated over wireless links. A range of technologies is available for extra-BAN communication including Bluetooth, WLAN, GSM, GPRS and UMTS.

When the devices of a BAN measure perform actions for healthrelated purposes we call this kind of specialization of Body Area Networks a health BAN. As a further specialisation, a BAN whose devices are biosignal sensors enables patient monitoring. We can envisage any number of health monitoring BANs for specific clinical conditions. Some example applications are: cardiac care, diabetes management, sleep analysis, asthma monitoring, epilepsy monitoring, SIDS, movement analysis and monitoring and control of implanted drug delivery systems. If we combine health BANs with extra-BAN communications, this enables a range of distributed applications including remote monitoring of patients by health professionals. 
The MobiHealth BAN consists of a central unit known as the MBU (Mobile Base Unit) and a set of body-worn devices, which may include sensors, actuators, and various multimedia devices. In principle the BAN may incorporate any wearable or implantable devices. Figure 1 shows the MobiHealth BAN and its relation to the $\mathrm{m}$-health service provider and the healthcare service provider.

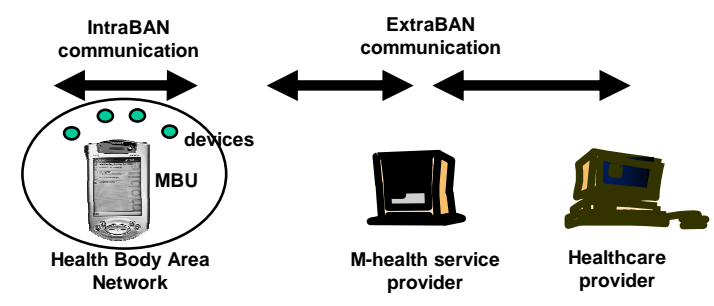

Figure 1. The MobiHealth Body Area Network

The BAN software or BANware is implemented on top of a Java virtual machine (J2ME, CDC and Personal profiles) running under Linux. The MBU platform is currently the HP iPAQ 3870. Future plans include porting to other platforms including cell phones (eg. Sony Ericsson P800).

The generic BAN can be customized with different device sets for different applications. Some of the BAN devices used during the MobiHealth project were: electrodes for measuring ECG and EMG, activity sensors, respiration sensors and pulse oximeters. Other BAN devices used were marker/alarms and digital cameras.

\subsection{The Back End System}

Deployed BANs are supported by a MobiHealth Back End System (BeSys)which mediates between end user applications in the healthcare provider domain (eg HISs) and the BANs. The BeSys may be located at a healthcare location such as a hospital or at another location entirely, eg at an m-health service provider site. The BeSys runs on a server and incorporates a Look Up Service (LUS) and hosts a surrogate which represents and manages the BANs in the field. The end-user application may consist simply of a viewer application or (in future) some much more complex suite of applications interfacing to the clinical systems of the healthcare provider.

\subsection{Communications}

The BAN has been implemented using both wired (front-end supported) sensors from TMSI and wireless (self-supporting) sensors from EISlab (Östmark et al, 2003). Both approaches use Bluetooth (IEEE 


\section{MOBIHEALTH: MOBILE SERVICES FOR HEALTH PROFESSIONALS}

802.15) for intra-BAN communication. The front-end also allows Zigbee (IEEE 802.15.4) as an alternative intra-BAN communication technology. For extra-BAN communication 2.5 and $3 \mathrm{G}$ public networks are used. The MBU communicates via Bluetooth to a GPRS or UMTS handset; from there the signals are transmitted to the remote healthcare location. The first UMTS (test) infrastructures became available in the Netherlands during 2003 and the trials were run over commercial GPRS services in all countries and over test UMTS infrastructures in the Netherlands, Spain and Sweden. A commercial UMTS service was launched in the Netherlands shortly after the completion of the trials.

\subsection{The m-health Service Platform}

Together the components described above comprise an m-health service platform. Figure 2 shows the functional architecture of the service platform.

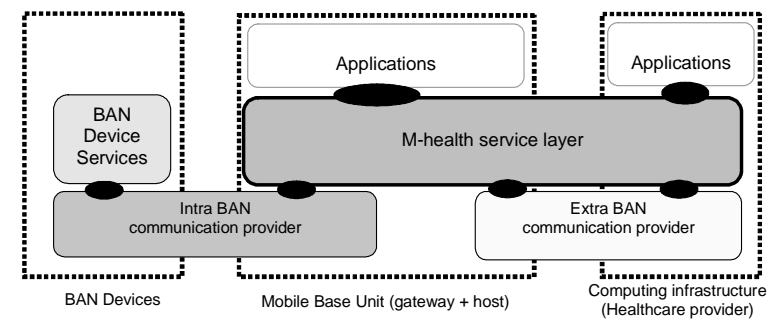

Figure 2. Service platform functional architecture

The m-health service platform supports the BAN based applications and isolates them from the details of the underlying communications services. It is the service platform which enables genericity and hardware independence. The services offered include BAN registration, discovery., security services, online configuration and management of BANs and BAN data storage. BAN data may be pulled by or punched to the remote application components. The BAN Interconnect Protocol (BANip) was implemented using HTTP1.1. The surrogate was implemented using the Jini Surrogate architecture. More details of the implementation may be found in the project deliverables, available at the project website and in (Dokovsky et al, 2003). The following section gives an overview of the MobiHealth services seen from the point of view of the health professional.

\section{PROFESSIONAL USE OF THE MOBIHEALTH SYSTEM}

The generic BAN concept and the generic m-health service platform permit any number of possibilities for mobile services for health professionals. Here we mention three classes of functionality for use by health professionals which have been explored in the course of the project. They are mobile monitoring systems, remote access to patients' 
BAN data, and mobile multimedia communication systems. These classes of functionality represent some of the possible configurations for a health professional BAN.

\subsection{Mobile monitoring system}

A BAN equipped with medical sensors can be carried by mobile health professionals so that they can perform various measurements on patients at the point of care in the community. The measurements can be viewed locally by the professional who applies the BAN to the patient. Furthermore the measurements can be transferred to a remote location over a variety of wireless communication services. At the remote locations(s) the clinical information can be uploaded and stored; it may also be viewed remotely by other members of the medical team.

Technically the health professional mobile monitoring system is very similar to the patient-controlled telemonitoring BAN. The major difference is that in the former case the BAN is part of the mobile equipment carried and used by a mobile health professional. User interface and access control requirements vary between patient-controlled and health professional-controlled use of BAN based monitoring systems. For some patient applications it may be that no local display of biosignals is needed or appropriate. For professional applications local viewing is normally required and the display must be of adequate quality for clinical use in that setting. For some purposes a PDA display will suffice; for others a higher resolution device is required.

In the MobiHealth trials different configurations of the BAN monitoring system were used by visiting nurses and by ambulance paramedics. Two version of the nurse BAN were developed for the trials. The trauma patient BAN is designed to be applied by ambulance paramedics to patients in an emergency setting.

Home monitoring following discharge from hospital. In one of the Swedish trials (Home care and remote consultation for recently released patients in a rural area) the target group is patients with multiple chronic diseases including cardiac failure, diabetes and respiratory disability. The intention is to support patients living in remote rural areas following discharge from hospital. If the patient or their family have concerns about the patient's condition they can request a home visit from a nurse. The visiting nurse carries a nurse BAN on home visits. The BAN can be used to measure ECG, heart rate respiration rate and oxygen saturation. Blood glucose, NIBP (blood pressure) and respiration are manually input to the BAN by the nurse. At the same time the measurements are transmitted over UMTS or GPRS to a remote physician or to a registered district nurse (RDN). The intention is to support clinical decision making by distributed teams; here the remotely located health professional views the clinical data during the home visit and determines together with the visiting nurse whether the patient needs transfer by ambulance to hospital. The expected benefit is that the use of the BAN will reduce the number of cases where the patient is moved to hospital unnecessarily. 


\section{MOBIHEALTH: MOBILE SERVICES FOR HEALTH PROFESSIONALS}

Vital signs monitoring in emergency medicine. In one of the Dutch trials (the Trauma trial), ambulance paramedics make use of two kinds of BAN. The first is known as the trauma patient BAN and is applied by the paramedic to the patient at the scene of the accident. The BAN devices are sensors to measure oxygen saturation, ECG and respiration. If they have time, the paramedics can also make manual inputs (pupil reaction, fluids) to the BAN. During trials information was transmitted from the trauma patient BAN via GPRS and UMTS to the emergency surgical team at the receiving hospital.

The trauma scenario implies much stricter requirements on the health professional user interface and on transmission parameters. In a critical emergency scenario the front-line professional needs to work fast and it is even more important in this kind of setting that the technology does not impede or interfere with their work by demanding they do extra tasks or deal with clumsy equipment. For transmission, the wireless communication with the hospital needs to operate well not only from the static situation (the scene of the incident) but also from the moving ambulance during transfer of the patient. This means maintenance of communication despite a fast moving source which possibly passes through different areas of coverage, generating tougher communication requirements relating to robustness and quality of service and the possible need to perform seamless horizontal and vertical handovers. The expected benefits of transferring clinical information from the scene are improved clinical outcomes for patients due to an earlier informed, and hence better prepared, emergency department at the hospital.

The following section describes another BAN configuration which supports a different set of mobile services for health professionals. The variants of the BAN mentioned so far are all telemonitoring systems and as such are principally producers of data. Corresponding to the data producers are the data consumers which were originally conceived as applications running at a remote health care location such as a hospital or health call centre within the jurisdiction of the health service provider. Some of these applications will involve exchange of m-health data with the EMR and hospital information systems and as such are outside the scope of the current project. However some of the applications are appropriate to run on a health professional's mobile device, allowing the health professional to be fully mobile and to have ubiquitous access to BAN services and data. Examples explored during the project, and future planned extensions, are discussed below.

\subsection{Mobile access to patient BAN data}

Within the scope of the current project the end user applications developed for use by healthcare professionals consist of alarm services and applications to process and view data from patient's mobile telemonitoring systems.

Alarm services. If needed a patient BAN can be equipped with an alarm button which the patient can press to summon help. The alarm service is a real time service linking a (mobile) patient with a (mobile) 
health professional. In the current implementation the health professional receives the alarm notification by SMS message to a mobile phone.

Future developments could include more sophisticated versions of the alarm service. From the health professional point of view the reception of alarms could be one of the services with which the generic health professional BAN could be configured as appropriate. An important evolution would be the automatic detection of (clinical) emergencies and generation of alarms without patient intervention. Automated alarm services could range from simpler cases such as detecting when threshold values are exceeded to intelligent interpretation of multiple data sources such as activity sensors, biosensors and, possibly, environmental sensors together with patient specific information such as the EMR or an emergency dataset.

Viewer applications. A simple viewer application was implemented to run on the MBU. The viewer displays numerical values such as heart rate and $\mathrm{SaO} 2$ and visualisations for continuous signals such as ECG and pulse plethysmogram. The intention was that the viewer application should be used to verify that sensors are attached and functioning properly. However the graphical display and the numerical values can be used for some clinical purposes. For other clinical purposes better display capabilities and a more sophisticated viewer application is needed. In the trials the Portilab 2 viewer from TMSI was used. This system can be run on a health professional's laptop to give higher resolution and scalable displays. Portilab 2 running on a laptop with wireless internet access has already been tested as a mobile application for health professionals. Such applications are candidate services for the customizable health professional BAN.

Processing, analysis and interpretation of biosignals. Underlying the viewer applications are functions to process the raw sensor output. Signal processing functions (analogue to digital conversion, filtering and compression) are applied to the raw sensor data by the sensor system. Higher level applications perform further processing and analysis of incoming sensor data. An example is the derivation of calculated parameters from measured parameters (eg heart rate derived from pulse plethysmogram) or the separation of different signals (eg separation of foetal ECG from maternal ECG). These functions are distributed across the sensor system (the sensors themselves and the DSP in the sensor front end) and the Portilab 2 software.

For the future we can postulate a range of clinical interpretation functions involving sophisticated high level clinical inferencing systems applied to multiple biosignal streams sampled over time. Intelligent automated processing of BAN data is a prerequisite for large scale deployment of some telemonitoring services in the population, since continuous monitoring requiring observation of BAN output 24/7 by clinical staff would make impossible resource demands on health services. 


\subsection{Mobile Multimedia communication system}

Earlier we referred to the telemonitoring BAN used in the trauma setting. The other type of BAN used in the Trauma trial is the paramedic BAN. This has different kinds of functionality from the patient BANs and the nurse BANs already described. Rather than being applied to a patient by a health professional this kind of BAN is worn by the health professional and in this case the BAN devices are multimedia devices. The purpose is to provide real time communication between the paramedics and the team at the hospital. The original vision was to equip members of the distributed trauma team with health professional BANs enabling high quality hands free audio and video communication between the paramedics and the hospital staff.

At time of writing the paramedic BAN consists of an MBU and one device: a Bluetooth enabled digital camera; hence visual communication only is supported. Still images from the scene are transmitted from the camera to the MBU via Bluetooth and to the hospital over GPRS or UMTS. When digital video cameras conforming to the Bluetooth video profile become available (expected 2004) the still images will be replaced by motion video. As with the trauma patient BAN, the paramedic BAN exchanges information with the hospital both from the scene and from the moving ambulance.

In future the intention is that by combining information from the paramedic BAN and the trauma patient BAN, the hospital team can participate through telepresence and, by means of augmented reality style presentation, be able to simultaneously view the patient, the scene of the accident and visualisations of the patient's biosignals.

The paramedic BAN and the Nurse BANs each represent a selected set of mobile services for health professional use; that is they are different instantiations of the generic BAN. They are all supported by the generic service platform which can support mobile services customized to the particular needs of individual professionals and which can be used to link multidisciplinary teams of mobile health professionals enabling them to function as a virtual team.

\section{FUTURE CHALLENGES}

The vision of ubiquitous healthcare poses challenges to many disciplines, both technical and non-technical. Currently the major limiting factor for provision of mobile services seems to be the rather mundane but problematic issue of the limited battery life of mobile devices. Power management strategies can make some inroads here but a breakthrough in power technologies which can make a difference in order of magnitude is needed. Regarding communication technologies, even with UMTS we still suffer from limited bandwidth for some remote monitoring applications, such as full 12-lead ECG or EEG transmission. There are many software engineering issues arising in connection with 
intelligent adaptive applications and communications, complicated by cross-cutting issues of security, quality of service and scalability.

For health service providers, large scale usage of mobile services implies access to high performance distributed computing and broadband wireless communication resources. More radically it also implies a high degree of automation of clinical analysis and interpretation. For some applications automated analysis will involve complex real time pattern recognition and application of temporal abstraction in order to automate clinical interpretation. This in turn implies that BAN applications interface to large scale and comprehensive clinical knowledge bases and inferencing systems.

We expect that in the longer term the current style of implementation of (health) BANs will disappear, replaced by nanoscale devices and contactless sensing from the ambient intelligent environment. We call this vision the Ambient Intelligent BAN or AmI-BAN). AmI-BAN devices might be implanted rather than worn externally on the body or in clothing. These devices could include for example actuators to control drug delivery systems, nanomanipulators or neural stimulators interfacing directly to motor neurons.

The vision of the future depends on scientific research and technological advances not only in computing science but also from other technical disciplines including electrical engineering, physics and nanoscience. Wearable computing, nanotechnologies, contactless sensing, implantable devices and low-power or self-powered devices are some of the enabling technologies prerequisite to the realization of the vision. Together these multidisciplinary developments can open the way for seamless collaboration between BANs, PANs and the ambient intelligent environment.

Beyond the technical domains, experience indicates that critical success factors for take up of technical innovation include organisational and management issues concerning the impact on ways of working and business processes and trust and acceptance by professional users. Research in the areas of e-health and e-learning indicates that innovation is embraced by busy professional users only when they perceive immediate benefits.

Regarding the organisational aspects of healthcare, exploitation of a technical innovation may imply extensive and radical business process re-engineering of the national (or pan-European) healthcare system. Failure to account for this kind of impact has caused many health technology innovations to fail in the past. Unless business process reengineering is driven by and accepted by the stakeholders, including the patients, the different categories of health professionals and other involved professional groups, the payers (eg. health insurance companies) and the policy makers, the most promising health technology innovation is likely to fail. Even then, evolution goes more smoothly than revolution.

Economic effects can also spell failure for take-up of sound technical innovations. A comprehensive cost benefit analysis is prerequisite, but 


\section{MOBIHEALTH: MOBILE SERVICES FOR HEALTH PROFESSIONALS}

should take into account all the direct and indirect financial costs (including cost savings) and set them against the non-financial factors, that is the benefits (such as improved health outcomes or increased user satisfaction) and the disbenefits, for all the stakeholders involved. The social and ethical issues, and legal and regulatory factors, also need to be included in the analysis.

\section{CONCLUSIONS}

The MobiHealth project ran for 22 months from May 2002. The main development focus was on the architecture, design and implementation of an m-health service platform to support mobile services for patients. The objective was to test out the potential of 2.5 and $3 \mathrm{G}$ communications to support mobile services. In the relatively short time of the project the phases covered were: user requirements, design and implementation of a generic health BAN including several specialisations of the BAN, and architecting of a generic m-health service platform. In addition trials and evaluation were conducted. At the time of writing the results of the evaluation are being compiled. Regarding mobile services for the professional user, the experience has demonstrated that the generic MobiHealth concept can be applied for the mobile professional user as well as for the patient user.

Further development continues, targeted at a fully generic and evolvable m-health service platform supporting mobility for both patients and professionals and supporting personalised and evolvable services adaptable to the needs of different categories of users operating in dynamically changing contexts.

\section{ACKNOWLEDGEMENTS}

Our thanks go to the European Commission, who funded the MobiHealth project under the IST FP5 programme, and to the MobiHealth partners. Thanks are also due for the support of the University of Twente (EEMCS, CTIT, and APS group).

\section{REFERENCES}

van Dam, K, S. Pitchers and M. Barnard, 'Body Area Networks: Towards a Wearable Future', Proc. WWRF kick off meeting, Munich, Germany, 6-7 March 2001; http://www.wireless-world-research.org/.

Dokovsky, N., A.T. van Halteren, I.A. Widya, "BANip: enabling remote healthcare monitoring with Body Area Networks", In proceedings of IEEE Conference on scientiFic engIneering of Distributed Java applIcations (FIDJI 2003), Luxemburg, November 2003.

Jones, Val, Rob Kleissen, Victor V. Goldman (1999), Mobile applications in the health sector, Presentation at Mobile Minded Symposium, 22 September 1999, University of Twente.

Jones, Val, Mobile applications in future healthcare, Presented at CTIT Workshop, University of Twente, 8 February 2001. 
Jones, V. M., Bults, R. A. G., Konstantas, D., Vierhout, P. A. M., 2001a, Healthcare PANs: Personal Area Networks for trauma care and home care, Proceedings Fourth International Symposium on Wireless Personal Multimedia Communications (WPMC), Sept. 9-12, 2001, Aalborg, Denmark, http://wpmc01.org/, ISBN 87-988568-0-4

Jones, V. M., Bults, R. A. G., Konstantas, D., Vierhout, P. A. M,. 2001b, Body Area Networks for Healthcare, Wireless World Research Forum meeting, Stockholm, 17-18 September 2001; http://www.wireless-world-research.org/

Jones, Val, Richard Bults, Pieter AM Vierhout, Virtual Trauma Team, 2001c, Wireless World Research Forum meeting, Helsinki, 10-11 May 2001; http://www.wireless-worldresearch.org/

Konstantas, Dimitri, Val Jones, Richard Bults and Rainer Herzog, 2002a, MobiHealth Wireless mobile services and applications for healthcare, International Conference On Telemedicine - Integration of Health Telematics into Medical Practice, Sept. 22nd-25th, 2002, Regensburg, Germany.

Konstantas, Dimitri, Val Jones, Richard Bults, Rainer Herzog, 2002b, MobiHealth innovative 2.5 / $3 G$ mobile services and applications for healthcare, Thessaloniki, 2002.

MobiHealth, 2002; http://www.mobihealth.org

Östmark, Åke, Linus Svensson, Per Lindgren, Jerker Delsing, "Mobile Medical Applications Made Feasible Through Use of EIS Platforms", IMTC 2003 Instrumentation and Measurement Technology Conference, Vail, CO, USA, 20-22 May 2003.

A. Pitsillides, G. Samaras, M. Dikaiakos, E. Christodoulou, DITIS: Collaborative Virtual Medical team for home healthcare of cancer patients, Conference on the Information Society and Telematics Applications, Catania, Italy, 16-18 April 1999.

Rekhter, Y., B. Moskowitz, D. Karrenberg, G. J. de Groot, E. Lear et al., "Address Allocation for Private Internets", RFC 1918, February 1996.

Schmidt, R., 2001, Patients emit an aura of data, Fraunhofer-Gesellschaft, www.fraunhofer.de/english/press/md/md2001/md11-2001_t1.html

Widya, A. van Halteren, V. Jones, R. Bults, D. Konstantas, P. Vierhout, J. Peuscher, 2003. Telematic Requirements for a Mobile and Wireless Healthcare System derived from Enterprise Models. Proceedings IEEE ConTel 2003: 7th International Conference on Telecommunications, June 11-13, 2003, Zagreb, Croatia.

Wireless World Research Forum, 2001, The Book of Visions 2001: Visions of the Wireless World, Version 1.0, December 2001; http://www.wireless-world-research.org/

Zimmerman, T.G., 1999, 'Wireless networked devices: A new paradigm for computing and communication', IBM Systems Journal, Vol. 38, No 4. 